\title{
Coarse-grained molecular dynamics simulations of nanoplastics interacting with a hydrophobic environment in aqueous solution
}

\author{
Lorenz F. Dettmann ${ }^{a}$, Oliver Kühn ${ }^{a b}$, and Ashour A. Ahmed ${ }^{a b *}$
}

\begin{abstract}
Nanoplastics (NPs) are emerging threats for marine and terrestrial ecosystems, but little is known about their fate in the environment at the molecular scale. In this work, coarse-grained molecular dynamics simulations were performed to investigate nature and strength of the interaction between NPs and hydrophobic environments. Specifically, NPs were simulated with different hydrophobic and hydrophilic polymers while carbon nanotubes (CNTs) were used to mimic surface and confinement effects of hydrophobic building blocks occurring in a soil environment. The hydrophobicity of CNTs was modified by introducing different hydrophobic and hydrophilic functional groups at their inner surfaces. The results show that hydrophobic polymers have a strong affinity to adsorb at the outer surface and to be captured inside the CNT. The accumulation within the CNT is even increased in presence of hydrophobic functional groups. This contribution is a first step towards a mechanistic understanding of a variety of processes connected to interaction of nanoscale material with environmental systems. Regarding the fate of NPs in soil, the results point to the critical role of the hydrophobicity of NPs and soil organic matter (SOM) as well as of the chemical nature of functionalized SOM cavities/voids in controlling the accumulation of NPs in soil. Moreover, the results can be related to water treatment technologies as it is shown that the hydrophobicity of CNTs and functionalization of their surfaces may play a crucial role in enhancing the adsorption capacity of CNTs with respect to organic compounds and thus their removal efficiency from wastewater.
\end{abstract}

\section{Introduction}

Plastics are synthetic or semi-synthetic organic materials that contain mostly polymers as the main component. They are lightweight, corrosion-resistant, and inexpensive materials with a wide range of applications. The downside of their durability are the problems associated with plastic waste and plastic debris in the environment. Plastic debris can disintegrate into smaller particles, even under ambient conditions. ${ }^{1]}$ Plastic particles that are smaller than a few millimeters in size are commonly known as microplastics and particles smaller than $1000 \mathrm{~nm}$ are known as nanoplastics (NPs). ${ }^{[2}$ These particles are widespread in the environment, including the oceans $s^{3}$ and freshwater in Europe, 4 North America, ${ }^{5}$ and Asia ${ }^{6}$. In marine ecosystems, plastic waste do not only cause aesthetic problems, but it can be also digested by organisms that mistake it for food. $780 \%$ of the plastics in the marine wastes come from land. ${ }^{[8}$ It was estimated that there may be 4 to 23 times more microplastics on land than in the ocean and that soil alone may contain more microplastics than the oceans. 9 In terrestrial systems, a primary entry point for microplastics is the agroecosystem, which is connected to the wide application of plastic mulch and sewage sludge. $10-13$

In agricultural ecosystems, various noxious substances, like persistent organic pollutants, or heavy metals can be adsorbed on plastic particles. Thereby, plastic particles can act as vectors for such environmental contaminants. ${ }^{14}$ In general, transformations of NPs via homo- and hetero-aggregation could lead to advective flow transport, sedimentation, photo- and biodegradation, and/or sediment entrapment might occur. $15 / 16$

${ }^{a}$ University of Rostock, Institute of Physics, Albert-Einstein-Str. 23-24, D-18059 Rostock, Germany. E-mail: ashour.ahmed@uni-rostock.de

${ }^{b}$ University of Rostock, Department of Life, Light and Matter (LLM), Albert-Einstein-Str. 25, D-18059 Rostock, Germany
Estimation of NPs in the environment requires very sensitive and selective analytical techniques to detect the different types of NPs. These techniques would have to be sensitive towards particle sizes in the nanometer range and to concentrations down to nanograms per liter. Regarding the NPs investigation in soil, none of the published methods, such as vibrational spectroscopy or chromatography seems to be ideally suitable to detect these very small plastic particles. Regarding the spectroscopic measurements, the background fluorescence of organic matter or pigments of some polymers can strongly interfere with the spectra of interest, making them unidentifiable. ${ }^{17}$ Moreover, soil components can form relatively stable aggregates which may enclose plastic particles.18 Ongoing modifications and combinations of methods, however, may help to enhance the currently very limited data in this field of research. 19

Carbon nanotubes (CNTs) are macromolecules formed by sheets of graphene. They are characterized by a very high surface area, low density, high mechanical resistance, strong hydrophobicity and severe biodegradation. ${ }^{20}$ Due to their unique physicochemical properties, CNTs have a wide range of technological applications. ${ }^{21}$ This includes their use in the fields of water treatment and agricultural sector. In agricultural, CNTs were used as nanosensors for various tasks involving detection and release of toxic substances to regulate plant growth and also to increase the life of fruits after harvest. ${ }^{[22-27}$ But also some toxic effects have been recorded for CNTs in soil and specifically in the agricultural sector. 2829

In a more fundamental study Hyung and Kim 30 investigated the adsorption behavior of standard humic and fulvic acids to multi-walled carbon nanotubes (MWCNTs). Based on the Freundlich model, they found that there is a positive linear correlation between adsorption capacity and amount of aromatic group content. Ateia et al. ${ }^{[31}$ studied the adsorption process of the same humic and fulvic acids on MWCNTs and also found a linear cor- 
relation between adsorption constants and aromaticity (aromatic content) of a specific molecular system. In addition, they pointed to the dependence of the adsorption on the degree of oxidation and carboxylic acidity of the considered organic molecules. In a metastudy Engel and Chefetz ${ }^{32}$ evaluated a large set of data to quantitatively validate the correlation between aromatic content of dissolved organic matter and its adsorption to CNTs. The result has been negative, i.e. no correlation was observed. This contradiction with earlier work on specific cases was explained by the complexity and heterogeneity of dissolved organic matter and different CNTs characteristics.

While there is a huge number of literature covering interactions between NPs ${ }^{33|35|}$ or CNTs $30|31| 36|38|$ with organic compounds, no studies are addressing the interaction between NPs and CNTs, although both chemical compounds might coexist, especially in the terrestrial environment. Moreover, CNTs provide a simple model system for studying the interaction of NPs with hydrophobic surfaces and cavities, the latter being an abundant motif in macromolecular soil organic matter particles. $39 \sqrt{40}$ Specific functional groups present in these macromolecules can easily be added to the CNTs and their effect, e.g., on NPs binding can be quantified. Therefore, our objective is to introduce a molecular level understanding for the interaction of NPs with CNTs, which not only addresses this particular system but it should also mimic aspects of the interaction of NPs with soil organic matter. It should be noted that polymers in confined environments were investigated extensively throughout the last years. 41,47 Such models are not only a classical problem in polymer physics 48 , but also of interest because of their connection to biological processes like DNA packing in capsids 49 or chromosome separation during cell division 50 . In most of the investigations, entropic effects, chain formation or segregation of multiple chains are in the main focus.

In the present contribution coarse-grained molecular dynamics (CGMD), as an effective approach to describe polymers and CNTs, 51,54 will be used to model and simulate the behavior and interactions between NPs (unbranched polymer chains) and CNTs (bare and decorated with functional groups) in the presence of water. The rest of the paper is organized as follows. A detailed description of the modeling approach and computational details is presented in the next section. Then, the simulation results of different model systems are discussed. Finally, conclusions concerning the most important outcomes are presented.

\section{Methods}

\subsection{Molecular Modeling}

The present study focuses on three different model systems. First, NPs in water will be studied to establish a relation between the number of monomer units and the radius of gyration in absences of CNTs. Second, the CNT is included to investigate its interaction with the NPs. Third, the CNTs are functionalized with organic groups to investigate the effect of the modified hydrophobicity of CNTs on the interaction between NPs and CNTs.

\subsubsection{NPs in water}

Polyethylene (PE), polypropylene (PP) and polystyrene (PS) are among the most abundant macro- and micro-plastics in marine and soil ecosystems. 11:55 59 Therefore, in the present study we modeled NPs by considering PE, PP and PS. Furthermore, polyethylene oxide (PEO) as a hydrophilic polymer was considered as well to take into account the hydrophobicity/hydrophilicity effect. Specifically, NPs were modeled as unbranched polymer chains, based on the MARTINI model for polymers. 60 63 The mapping schemes between the atomistic and the coarse-grained representations are introduced in Fig. 19). For every polymer type, chains with different number of monomers $N$ were considered, ranging from 5 up to 45 (for PEO), 200 (for PE), 350 (for PP) and 114 (for PS). These maximum chain lengths cover approximately the same range for the radius of gyration, $R_{\mathrm{g}}$. Polymers were put into the center of cubic boxes. Each simulation box contains one single polymer. The length of one box vector was chosen depending on the number of monomers for each polymer: $50 \AA$ for $N \leq 10,100 \AA$ for $N \leq 30$ and $150 \AA$ for $N>30$. The boxes were filled with water particles with a density of about $1 \mathrm{~g} \mathrm{~cm}^{-3}$ using the Packmol software. 64 In the MARTINI model one particle combines four atomistic water molecules.

\subsubsection{NPs with CNTs in water}

To explore the interaction between NPs and CNTs, different setups involving either PEO, PE, PP and PS of the previous section and a single CNT in water were considered. The coarse-grained CNT model was constructed by the "Open Carbon Nanotubes for the MARTINI Model" tool. $[55$ Exemplarily, a length of $100 \AA$ and a diameter of $30 \AA$ was chosen. A comparison of the coarsegrained and atomistic representation is shown in Fig. 1p). For each NP-CNT model, CNT was placed at the center of the simulation box, with box dimensions of $140 \AA \times 140 \AA \times 240 \AA$. Afterwards, one polymer was added into the constructed box. Then, the box containing the NP-CNT molecular system was filled with water, having a density of about $1 \mathrm{~g} \mathrm{~cm}^{-3}$. For each NP-CNT model, each polymer had two different initial configurations. First, the polymers were placed inside the CNT at approximately one quarter of the CNT's length (see Fig. 1 1 )). Second, the polymers were placed in water at about $20 \AA$ distance from the outer wall of the CNT (see Fig. 1 d)). For each polymer, different chain lengths were considered based on their average $R_{\mathrm{g}}$ values in water, i.e. 6 , 7, 8, 9, and $10 \AA$. With four different polymers (PE, PP, PS and $\mathrm{PEO}$ ), five different chain lengths and two starting configurations for each chain, in total, $40 \mathrm{NP}-\mathrm{CNT}$ systems were investigated. All initial configurations were chosen in a way that the polymers go through configurations of interest with sufficient duration time.

\subsubsection{NPs with functionalized CNTs in water}

To modify the hydrophobicity of the CNTs of the previous section, functional groups were introduced by attaching them to the inner walls of CNTs. The functional groups were inserted at the center of CNTs and kept fixed in position. Here, three different abundant soil organic matter functional groups are modeled, namely the nonpolar alkane chain (A) and phenyl group (PH) as well as the polar carboxylic acid group (CA). 66 Three groups of each 
a)

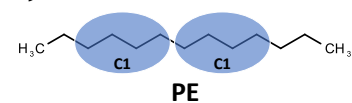

PE

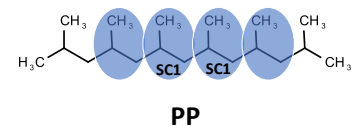

b)

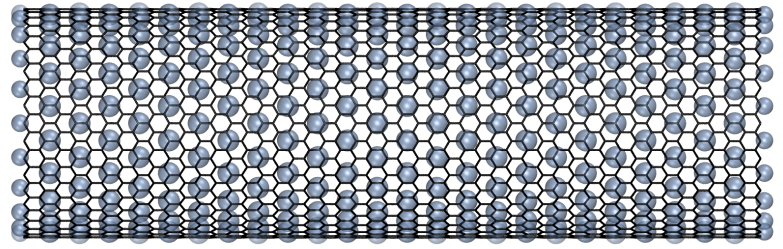

c)

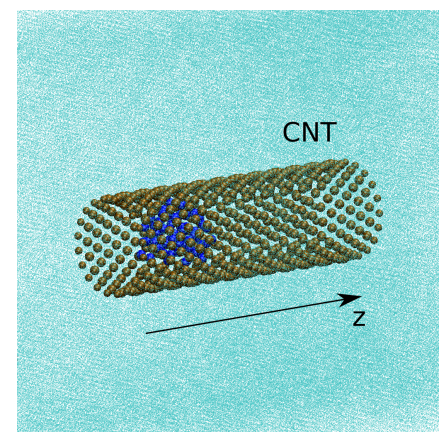

e)

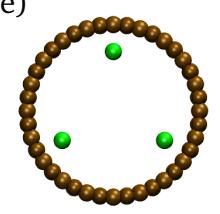

d)

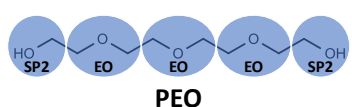

PEO
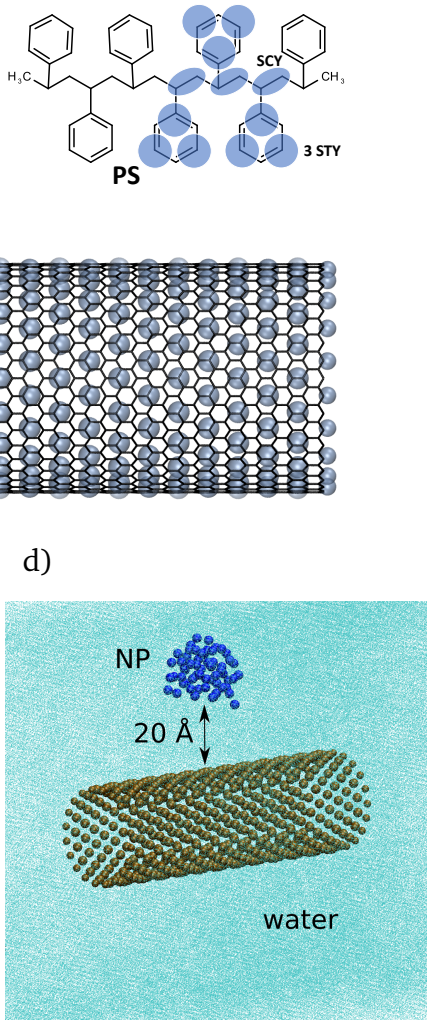

Fig. 1 a): Illustration of PE, PEO, PP and PS models. The black lines correspond to the atomistic and the blue circles to the coarse-grained representation of the polymers. b): Atomistic (black) and coarse-grained (blue) representation of the CNT. Two different starting configurations for PE, inside (c)) and outside (d)) the CNT. e): Front view of CNTs with three functional groups, placed in the middle of these CNTs. The green beads (left) represent alkane chains $(A)$, the pink beads (middle) represent carboxylic acid groups (CA) and the purple beads (right) represent phenyl groups (PH).

type were added to the CNT (see their arrangement in Fig. 19). To keep the model simple only one type of functional group was considered at the same time and all three functional groups are symmetrically arranged. For each polymer type, the case $R_{\mathrm{g}}=7 \AA$ was selected. For this value motion of the polymer through the CNT would be possible, at least in principle. For each NP-CNT system, polymers were initially placed inside the CNT at one quarter of its length. The boxes of NP-CNT systems were filled with water, with the same water density, and box dimensions as used in the previous section. Eventually, with four different polymers (PE, PP, PS and PEO) and three different functional groups (A, CA and PH), 12 NP-CNT systems were considered.

\subsection{Computational details}

MARTINI force field $\sqrt{60}$ based coarse-grained molecular dynamics simulations were performed using the GROMACS software package (version 2019.04). ${ }^{72}$ After energy minimization, an NPT simulation of $100 \mathrm{~ns}$ was done to adjust the box dimensions. In the latter step, the polymers were kept fixed at their initial position. Production runs were carried out with $N V T$ molecular dynamics simulations for $500 \mathrm{~ns}$ for each setup. Here, the CNT was kept fixed at its initial position. The time step was set to $20 \mathrm{fs}$. In combination with the Verlet neighbor list scheme, a straight cutoff with a cutoff-distance of $11 \AA$ was used. Furthermore, the temperature was controlled with the velocity-rescale thermostat ${ }^{73}$ using a coupling constant of $1 \mathrm{ps}$. To control the pressure in the NPT simulations, the Berendsen barostat with a coupling constant of 12 ps was applied. Furthermore, the compressibility of the system was set to $3 \times 10^{-4} \mathrm{bar}^{-1}$. Freezing of water is a common problem in coarse-grained simulations. 60 T4/75 To overcome this problem and decrease the probability of freezing, antifreeze (AF) particles with concentration of $15 \%$ with respect to water particles were considered. In addition, a temperature of $310 \mathrm{~K}$ was used for the simulations in the NPT and NVT ensembles. The issues related to water freezing and diffusion of water through CNTs with different lengths and diameters were considered in the $\mathrm{ESI} \dagger$ (see section I involving Table S1 and Figs. S1-S2).

The radius of gyration, $R_{\mathrm{g}}$, is defined as the mean-squared distance of the polymer beads to their center of mass, $\boldsymbol{r}_{G}$, i.e.

$$
R_{\mathrm{g}}^{2}=\frac{1}{M} \sum_{i=0}^{N} m_{i}\left\langle\left(\boldsymbol{r}_{i}-\boldsymbol{r}_{G}\right)^{2}\right\rangle
$$

where $m_{i}$ and $\boldsymbol{r}_{i}$ are the mass and position of the polymer bead $i$ respectively, and $M$ is the total mass of the polymer. $R_{\mathrm{g}}$ was calculated using the VMD visualization program (version 1.9.3) 76 . Note that the average $R_{\mathrm{g}}$ values of the different polymers studied below do not exceed $R_{\mathrm{g}} \sim 15 \AA$ which is much smaller than the simulation box dimensions, thus spurious effects due to the periodic boundary conditions can be excluded.

To characterize the strength of interaction/binding of NPs (polymers) to non-polar environment (CNT), interaction energies $E_{\text {int }}$ between polymers and the rest of the system (CNT and water) were calculated as the sum of the Lennard-Jones interactions:

$$
E_{\mathrm{int}}=\sum_{i<j} 4 \varepsilon_{i j}\left[\left(\frac{\sigma_{i j}}{\left|\boldsymbol{R}_{i j}\right|}\right)^{12}-\left(\frac{\sigma_{i j}}{\left|\boldsymbol{R}_{i j}\right|}\right)^{6}\right]
$$

These energies were calculated via energy groups using GROMACS tools. The cutoff for the Lennard-Jones interaction was set to $11 \AA$, which is the standard cutoff used in the MARTINI force field.For the present models, no charged beads were applied. However, it should be emphasized that the electrostatic interactions are involved implicitly within the Lennard-Jones parameters and especially via the interaction strength $\varepsilon .60$ In principle, 10 different interaction levels among polar, non-polar, apolar and charged molecular systems or moieties could be defined based on the $\varepsilon$ parameter. GROMACS tools were also used to calculated the partial density profiles of the NP particles along the $z$-dimension of CNT (see Fig. 1 k) and d)). Given the full density 
$\rho(\mathbf{r})$, the partial density is defined as

$$
P(z)=\iint \rho(\boldsymbol{r}) \mathrm{d} x \mathrm{~d} y
$$

For a better comparison between the different polymer types, the resulting partial density distribution $P(z)$ was normalized by its numerically calculated integral along the CNT:

$$
\tilde{P}(z)=\frac{P(z)}{\int P(z) \mathrm{d} z}
$$

This quantity can be interpreted as a position probability density.

\section{Results and Discussion}

The NPT equilibration simulations produced a density of around $0.86 \mathrm{~g} \mathrm{~cm}^{-3}$ for each investigated system. This deviation mainly comes from the usage of antifreeze-particles. It is a known effect and is caused by the stronger repulsion between antifreeze and water particles. It is assumed that models are still realistic enough to represent a system at nearly room temperature. It should be noted that all analyses are done with considering only the $N V T$ production trajectories.

\subsection{NPs in water}

The dependence of the radius of gyration, Eq. (1), on the number of monomers $N$ is shown in Fig. 2 for each polymer. Physical prop-

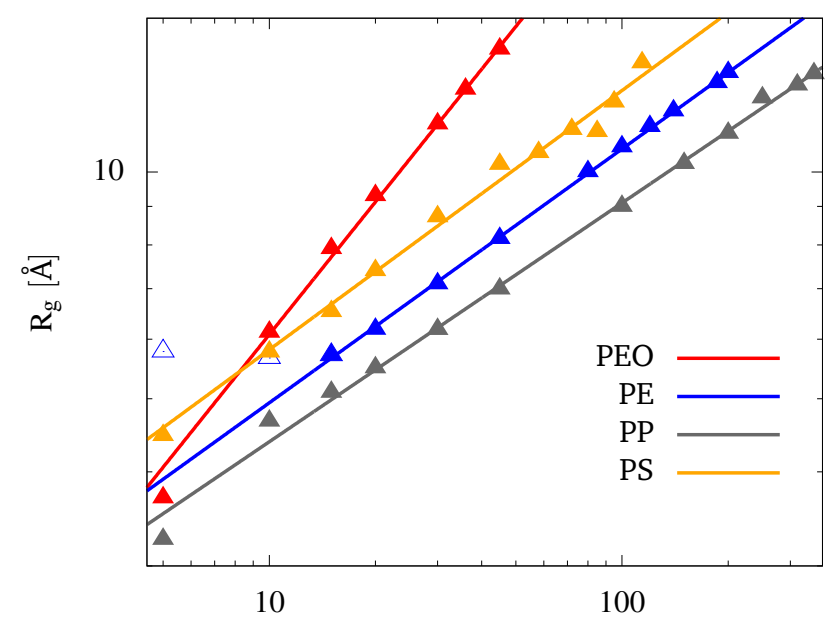

number of monomers $N$

Fig. 2 The dependence of the radius of gyration, $R_{\mathrm{g}}$, on the number of monomers, $N$, for the polymers in water (symbols). Also shown is the fit to Eq. 5 (straight lines). For PE, the first two data points are excluded from the fit.

erties of the polymers such as hydrophobicity and hydrophilicity can be read off from Fig. 2. For PEO, $R_{\mathrm{g}}$ for a given chain length is larger than for the other polymers. This points to the PEO's hydrophilic nature leading to a more unfolded structure as compared to the hydrophobic PE, PP, and PS which tend to minimize their contact area with a polar solvent. However, one should not take the overall lowest $R_{\mathrm{g}}$ values for PP as an indication of its largest hydrophobicity. Instead, one should also take into account the relatively short bond length and a low mapping compared to e.g. PE (see Table S2 in the ESI $\dagger$ ). PS, on the other hand, has also a comparable short bond length between beads of its backbone, but shows the largest $R_{\mathrm{g}}$ from the hydrophobic polymers. This is due to the phenyl groups, that increase the occupied volume of the folded PS chain and therefore raise $R_{\mathrm{g}}$. The degree of hydrophobicity was estimated by calculating interaction energies and radial distribution functions between NPs and water (see Figs. S3-S4 in the ESI $\dagger$ ). From the interaction energy values, one can conclude that PE has the highest hydrophobicity, while PS has the lowest one among the considered hydrophobic polymers.

According to the Flory theory of polymers, $R_{\mathrm{g}}$ is proportional to the number of monomers to the power of the so-called Flory exponent $v 48$. Therefore, the fit function

$$
R_{\mathrm{g}}(N)=a \cdot N^{v}
$$

was used to fit the data points shown in Fig. 2 and to determine the Flory exponent of each polymer type. Application of the Flory model to coarse-grained polymers is problematic for small chain lengths, where the discretization of the chain has a larger influence on the polymers behavior than the interacting with the solvent. In the present case, this is particularly prominent for PE whose coarse-grained representation has the longest bond length as compared to the polymers (see Table S2 in the ESI $\dagger$ ). Therefore, the first two data points have been excluded form the fit. For the remaining data points the Flory model is clearly confirmed $\left(R^{2}=0.99\right.$ in all cases). The calculated fit parameters are shown in Table 1 .

Table 1 Calculated fit parameters according to the mean field approach of Flory, for each polymer type. $v$ is the Flory exponent.

\begin{tabular}{lllll}
\hline & PEO & PE & PP & PS \\
\hline$a[\AA]$ & 1.58 & 2.27 & 2.11 & 2.63 \\
$v$ & 0.59 & 0.34 & 0.32 & 0.34 \\
\hline
\end{tabular}

The Flory exponent makes a statement about the polymersolvent interaction, especially whether the polymer is in a bad or good solvent. As water is polar, it is a good solvent for PEO and a bad solvent for PE, PP and PS. This can be observed from the calculated Flory exponents. According to the Flory theory for polymers, the Flory exponent is around 0.59 , if a polymer is present in a good solvent, while it is around 0.33 if a polymer is present in a bad solvent. From the almost quantitative agreement observed in Table 1 one can conclude that the present coarse-grained model provides a reliable representation of the polymer-solvent interaction. There has been one previous study of PEO in water using the MARTINI force field by Grunewald et al. 61 in which they obtained $a=1.81$ and $v=0.58$. The larger value for $a$ is likely due to the different temperature $(298.15 \mathrm{~K})$ and antifreeze particle concentration $(\mathrm{AF}=10 \%)$ used by these authors. 


\subsection{NPs with CNTs in water}

\subsubsection{Trajectory analysis}

In the following, interaction energies are calculated and analyzed in terms of polymer configuration and positioning, i.e. in solution as well as at the outer surface and inside the CNT. These positions are realized by choosing different initial conditions as shown in Fig. 11.) and d). One should notice, that some polymers (mostly PEO chains) move inside the CNT, which had a starting configuration outside. In the following, results of systems with polymers having radius of gyration $R_{\mathrm{g}}$ of $9 \AA$ in water and with initial conditions outside the CNT are summarized. An example for inside initial conditions for PE is given in Fig. S5 in the ESI $\dagger$.

During the trajectory PEO is in a relatively unfolded state (this holds for all $R_{\mathrm{g}}$ values used in this study) with an $R_{\mathrm{g}}$ value not much different from the solution case, see upper panel of Fig. 3 . PEO partly sticks at the outer CNT wall (cf. middle snapshot), but frequently goes back into solution and returns. Once inside the CNT, PEO prefers to stay directly attached at the inner wall. Furthermore, some polymers go through every of the three introduced positions in one trajectory (in solution, outer walls, inside CNT).

The interaction energies along the trajectory are shown in Fig. 3 as well. Notice that due to the cutoff for the Lennard-Jones potentials ( $11 \AA$ ) no interaction with CNT would be observed when $\mathrm{PEO}$ is far away from the CNT in solution. However, from the red curve in the upper panel of Fig. 3 we notice that the interaction energy is nearly unchanged along the trajectory, no matter whether PEO is in solution or at the outer wall of the CNT. Once PEO is inside the CNT, the interaction is slightly decreased. Note that in this work, more negative $E_{\text {int }}$ implies a stronger interaction. Thus we conclude that the interaction between PEO and the CNT is not much different than with water. The similarity of the two environments is also reflected in the almost unchanged radius of gyration which fluctuates around $9 \AA$. Of course, for the configurations inside the CNT once should note that $R_{\mathrm{g}}$ is much smaller than the CNT's diameter such that PEO does not have to make significant structural adjustments to go through the CNT.

The situation is rather different for the hydrophobic PE as shown in the lower panel of Fig. 3. In solution PE folds into a sphere like object. In contrast to PEO, once a PE chain adsorbs to the outer wall of the CNT, it stays there and does not go into solution again. Being at the outer wall, two configurations can be distinguished, i.e a folded (A) and an unfolded one (B). The two configurations can be separated from one another by their different interaction energies and $R_{\mathrm{g}}$ values as seen in Fig. 3

Regarding the interaction energy between $\mathrm{PE}$ and the rest of the system the blue curve shows its course of time for sticking in an outer wall position. When the polymer is adsorbing at the CNT in the A configuration, the interaction energy drops to around $-600 \mathrm{~kJ} \mathrm{~mol}^{-1}$. When the polymer is changing to configuration $\mathbf{B}$, the interaction energy drops further by $-350 \mathrm{~kJ} \mathrm{~mol}^{-1}$. This increased interaction is due to the increase of the contact area with the hydrophobic CNT as well as with the hydrophilic water in the unfolded state.

Compared to PEO, the interaction of PE with the CNT is much

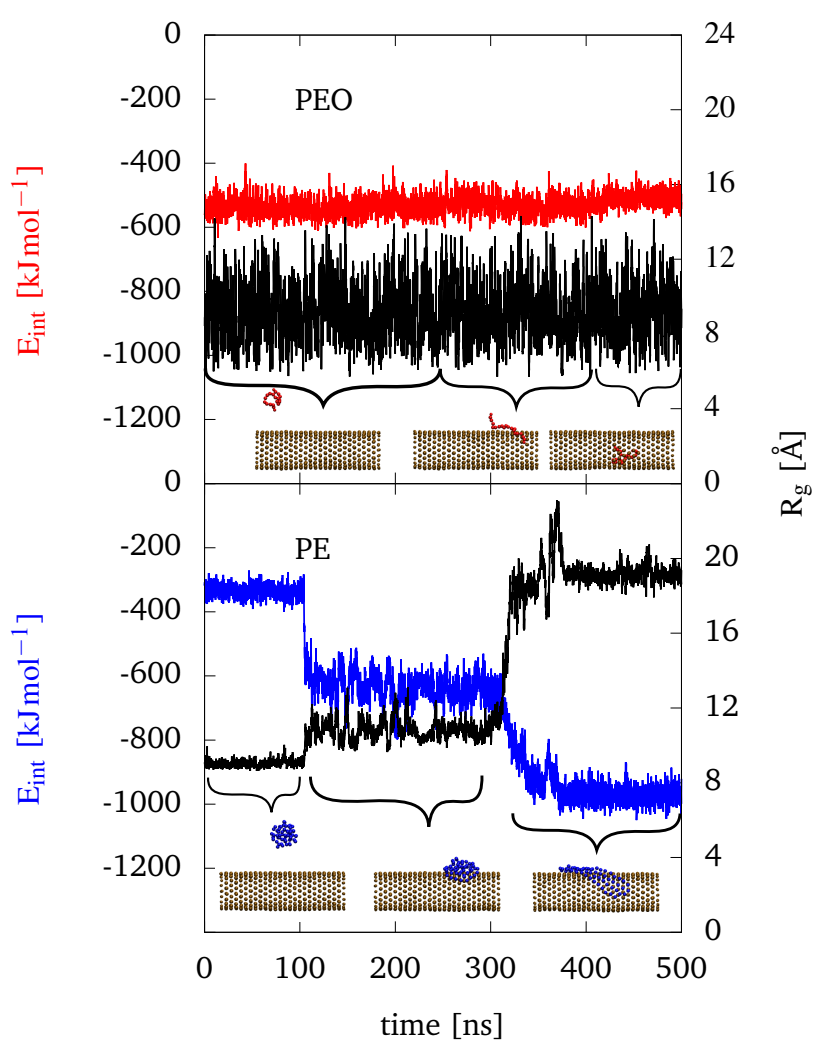

Fig. 3 Interaction energies (red and blue) along the production trajectories for PEO and PE with initially $R_{\mathrm{g}}=9 \AA$. For PE, the line relates to the simulation with the polymer outside the CNT. The interaction energy between the respective polymer and the rest of the system is considered. Furthermore, $R_{\mathrm{g}}$ (black) is plotted for both polymers. The curly brackets denote the time intervals, in which position in the system the respective polymer is found. (For PEO: in solution, at the outer and inner wall of the CNT. For PE: in solution, at the outer wall in configuration $A$ and at the outer wall in configuration B.)

stronger and therefore leads to a more stable adsorbed configuration. Furthermore, the difference in positions (in solution or interacting with the $\mathrm{CNT}$ ) are more pronounced for PE. As a consequence PE doesn't change positions (solution, in/outside), in contrast to the hydrophilic PEO.

The differences between configuration $\mathbf{A}$ and $\mathbf{B}$ for PE are also visible in the time evolution of $R_{\mathrm{g}}$ in Fig. 3. For PE being in solution, $R_{\mathrm{g}}$ is around $9 \AA$. Then, after adsorbing at the CNT in configuration $\mathbf{A}$, it is fluctuating around $11 \AA$ and when the PE chain is unfolding itself into configuration $\mathrm{B}, R_{\mathrm{g}}$ increases to around $19 \AA$. So, PE maximizes the contact area to the CNT, which significantly increases $R_{\mathrm{g}}$ and also the interaction energy, as discussed above.

Regarding the other two hydrophobic polymers, PP and PS, the following observations can be made from the trajectory data shown in Fig. S6 in the ESI $\dagger$. Qualitatively, PP behaves like $\mathrm{PE}$, e.g. there are similar configurations $\mathbf{A}$ and $\mathbf{B}$ although PP is slightly less unfolded in case $\mathbf{B}$ as compared with PE. In addition the energy drop when interacting with the CNT is generally weaker. In case of PS there has been a difference insofar as it doesn't unfold once adsorbed at the outer wall of the CNT (see 


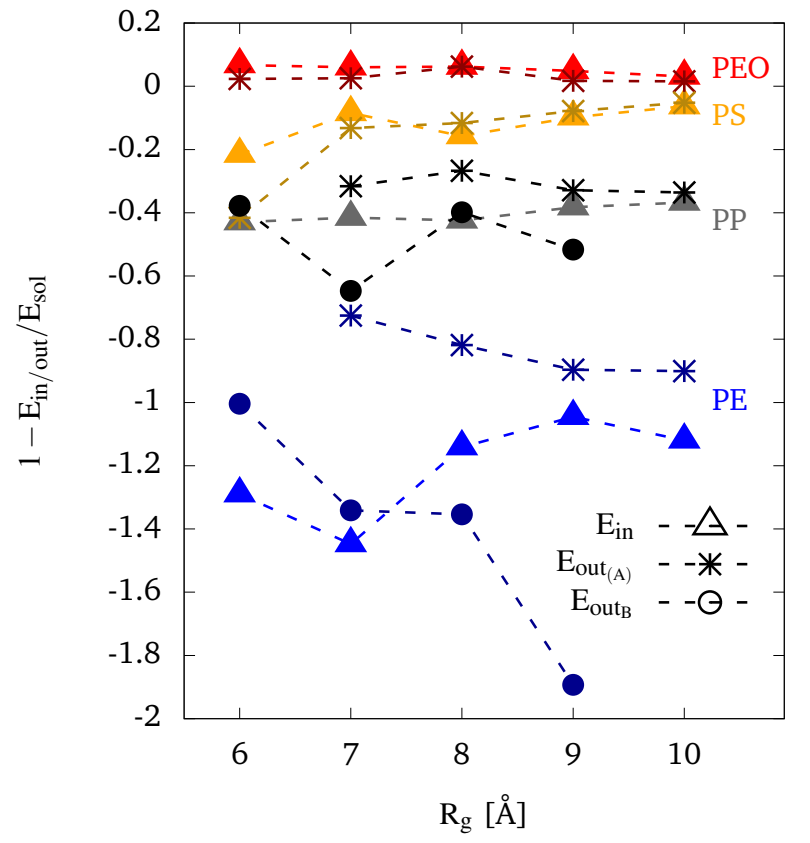

Fig. 4 Interaction energies relative to the bare solvent case as a function of solution phase radius of gyration and configuration. The colors stand for PEO (red), PE (blue), PP (gray) and PS (orange). For each polymer type, $\triangle$ denotes the interaction energy $\left(E_{\text {in }}\right)$, when the polymer is inside the CNT and $*$ refers to the interaction energy $\left(E_{\text {out }_{\mathrm{A}}}\right)$ at the outer wall of the CNT and for PE and PP, when the polymer is in configuration A. For $\mathrm{PE}$ and $\mathrm{PP}$, ○ denotes the interaction energy $\left(E_{\text {out }_{\mathrm{B}}}\right)$, when the chain is in configuration B. Cases corresponding to missing points were not observed in the production trajectory. For a better visualization data points are connected with dashed lines.

Fig. S6 in the ESI $\dagger$ ). The increase of interaction upon adsorption is relatively modest only. This can be explained by the less flexible structure of PS such that a smaller part of the polymer can directly attach to the CNT (change of $R_{\mathrm{g}}$ is negligible small). PS shows a slight decrease in the interaction energy with the rest of the system, when being adsorbed at the CNT. This change is less pronounced, when compared to the other hydrophobic polymers $\mathrm{PE}$ and PP, but larger compared to PEO. This behavior also reflects the relative strong interaction energy of PS to water (see Fig. S3 in the $\mathrm{ESI} \dagger$ ), compared to the other hydrophobic polymers.

\subsubsection{Interaction Energies}

In the following we will provide a more general picture of the interaction between the polymer and the rest of the system. It will cover $R_{\mathrm{g}}$ values (as obtained in solution, cf. Fig. 2) between $6 \AA$ and $10 \AA$. Interaction energies will be given relative to their values for the polymers in solution $E_{\text {sol }}$. Further, we will distinguish adsorption at the outer wall of the CNT ( $\left.E_{\text {out }}\right)$ and inside the CNT $\left(E_{\mathrm{in}}\right)$. For the case of outer wall adsorption we further differentiate between configurations $\mathbf{A}$ and $\mathbf{B}$ if applicable. The resulting $N V T$ averaged interaction energies are compiled in Fig. 4. In this diagram more negative values imply a high stability with respect to the polymer in solution.

For the case of PEO the relative interaction energies are above and close to zero for all chain lengths, no matter whether PEO is inside or adsorbed at the outer wall of the CNT. This confirms the conclusion drawn from Fig. 3 , i.e. that there is no preference for PEO to be adsorbed at the CNT. The positive values indicate its hydrophilic character.

The other extreme is $\mathrm{PE}$, which shows in particular noticeable differences with respect to the different configurations (cf. Fig. 3). Values for configuration $\mathbf{A}$ at the outer wall are around -0.8 , slightly decreasing with increasing $R_{\mathrm{g}}$. On the other hand, the relative interaction energies for configuration $\mathbf{B}$ are strongly decreasing with an increasing $R_{\mathrm{g}}$. This clearly indicates that with increasing polymer length, the gain in interaction energy due to the higher contact area in the unfolded state outperforms the interaction with water which dominate for the shorter polymers. PE inside the CNT does not show a clear trend, except a minimum at $R_{\mathrm{g}}=7 \AA$. PE (partly) unfolds inside the CNT. Given the size constraint due to the CNT, $R_{\mathrm{g}}=7 \AA$ is likely to present an optimum for unfolding and thus increasing the contact area. Compared to configuration $\mathbf{B}$ relative interaction energies for $\mathrm{PE}$ inside the CNT are lower or similar for a $R_{\mathrm{g}}$ up to $R_{\mathrm{g}}=7 \AA$, but higher for longer polymer chains. All values for PE inside the CNT are lower than for configuration A. Hence, we can conclude that for shorter chain lengths an inside configuration is preferred, whereas long chains tend to unfold in an outer wall configuration.

The behavior of the other hydrophobic polymers, PP and PS, is intermediate between the cases of PEO and PE. Looking at the values for PP, relative interaction energies in configuration $\mathbf{A}$ and inside the CNT are approximately constant, around -0.3 and -0.4 , respectively. For PP in configuration $\mathbf{B}$, similar or lower values (e.g. for $R_{\mathrm{g}}=7 \AA$ ) are observed, compared to the polymer being inside the CNT. Furthermore, configuration $\mathbf{B}$ results in lower values than configuration $\mathrm{A}$. This result is similar to $\mathrm{PE}$, although with a smaller gain in interaction energy. It agrees with the observations from the trajectories, namely that PP does not unfold itself that much when switching from configuration A to $\mathbf{B}$, compared to PE.

Regarding the relative interaction energies for PS, the values are approximately constant around -0.2 to -0.1 . Note that PS chains that were adsorbed at the CNT were observed in the folded A configuration only. An exception is the case $R_{\mathrm{g}}=6 \AA$ which could also have been counted as B configuration. This explains the deviation between interaction energies at this radius of gyration. Except for this case, all values for PS are higher than PP and lower than PEO.

\subsection{NPs with functionalized CNTs in water}

In the following we will consider the influence of organic groups placed inside the CNT (as shown in Fig. 1 1 ) ) on the binding of CNTs to NPs. The analysis will be performed in terms of the partial density $\tilde{P}(z)$ according to Eq. 5. Partial densities for all polymers (with $R_{\mathrm{g}}=7 \AA$ ) and different functionalizations are plotted in Fig. 5. Specifically, partial density profiles are calculated along the $z$ axis of the CNT (see Fig. 1 f)). Here, $z=0$ refers to the center of the CNT which the functional groups are placed as well. For each NP-CNT model with a specific functional group, only one simulation was performed by a starting conformation in which 


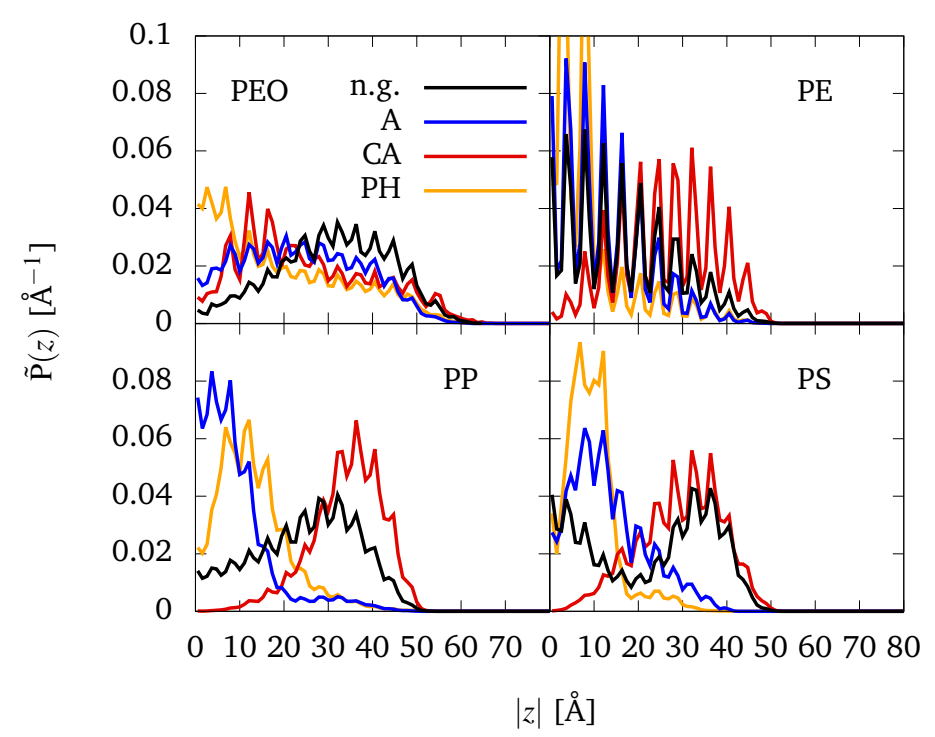

Fig. 5 Normalized partial densities, $\tilde{P}(z)$, for different polymers being (mostly) inside the CNT $(|z|<50 \AA)$, depending on the absolute value of the $z$-coordinate $|z|$. At $z=0$ the functional groups are situated, cf. Fig. 1). Four different cases are considered: no functional groups (black), alkane chains (blue), carboxylic acid groups (red), and phenyl groups (orange). Note that for PE-PH the two maxima which are out of the scale reach $\sim 0.16 \AA^{-1}$.

the polymer was placed at one CNT end (see 2.1.3). In principle, if the simulation started with a mirror configuration (i.e. the polymer was placed at the other CNT end), one would have a mirrored partial density profile. For this reason, in the following we are going to discuss the symmetrized partial density profiles, i.e. the normalized distribution $(\tilde{P}(z)+\tilde{P}(-z)) / 2$ along $|z|$. The complete partial density profiles along both negative and positive $z$ values are provided in Fig. S7 in the ESI†.

The distributions show more or less pronounced periodic modulations with a period of about $4.1 \AA$. This can be explained with the structure of the CNT which consists of rings of beads separated by about $4.07 \AA$, see Fig. 1 p). The beads of the polymers tend to be placed in between the CNT beads.

In case of PEO no significant differences are visible between the different setups. There is a tendency that a small part of the chain leaves the CNT, which points to slight preference of PEO to be surrounded by water molecules. Moreover, in the cases of alkane and phenyl functionalization, the polymer chain passes $z=$ 0 (see Fig. S7 in the ESI $\dagger$ ). At first glance it seems surprising that PEO accumulates at the phenyl groups. However, in the MARTINI force field the $\pi$ electron density of the phenyl rings is represented by a higher $\varepsilon$ value as compared to the alkane chain (2.66 vs 2.53). Note that the interaction area with a phenyl group is also higher, because they are described by three beads. Further note that the interaction with the carboxylic acid groups is masked by the presence of water, attracted to that group.

The distributions for PE do show significant differences with respect to the four setups. PE stays away from the center to avoid close contact with the hydrophilic carboxylic acid groups. In contrast the hydrophobic alkane chains and phenyl groups attract
PE which therefore shows a higher density towards the middle of the CNT as compared to the bare CNT case. Since the bulky phenyl groups present an obstacle for PE it cannot easily penetrate through $z=0$ and thus shows a higher density as compared with the case of alkane chains.

For the other hydrophobic polymers, PP and PS, the behavior concerning the hydrophilic carboxylic acid groups is similar to the case of PE. For PP and PS one notices that the oscillations are less pronounced due to the shorter bond lengths (see Table S2 in the ESI $\dagger$ ), i.e. its beads do not strictly fill out every of the spaces between the rings of the CNT. The same result can be obtained for PEO, also because it is less attracted by the CNT walls and therefore less influenced by its periodic potential. In absence of functional groups PP and PS tend to stay closer to their initial positions as compared to PE. On the one hand side, this looks reasonable considering the different bulkiness, cf. Fig. 19). On the other hand side, it could be that even the $500 \mathrm{~ns}$ trajectory doesn't fully sample the available configuration space.

In accordance with chemical intuition, the hydrophobic polymers have a preference for the hydrophobic functional groups and avoid hydrophilic ones. Water plays a role in controlling this behavior. Water particles are repelled by the hydrophobic functional groups, thus allowing the hydrophobic polymers to reach the region close the center more easily. On the other hand, water particles are attracted by carboxylic acid groups, making it harder for the hydrophobic polymers to access this region.

The structural changes of the polymers inside the functionalized CNT are addressed in Fig. 6. Here, $R_{\mathrm{g}}$ is plotted as a function of the distance between CNT and polymer centers of masses. Only the cases PE and PP are considered here, which according to Fig. 5 show pronounced differences (for PEO and PS, see Fig. S8 in the ESI $\dagger$ ).

For PE the mean values of $R_{\mathrm{g}}$ (black lines) for the systems with alkane chains and phenyl groups are higher than for the carboxylic acid and bare CNT cases. This increase in $R_{\mathrm{g}}$ correlates with the attraction to the hydrophobic functional groups. In all cases the polymer partly unfolds inside the CNT as compared to the solvated case $\left(R_{\mathrm{g}}=7 \AA\right)$. Concerning the spread of $R_{\mathrm{g}}$ values the bare CNT and the case of alkane chains do not behave much different. For the system with the phenyl groups, data points are less spread. This comes due to a arrangement of PE to a ring structure (see Fig. S9 in the ESI $\dagger$ ), in which it stays for more than the half of the simulation time. Also notable is the shift of the distribution towards larger $\delta_{\mathrm{CNT}-\mathrm{NP}}$ for the case of the carboxylic acid groups.

Compared to $\mathrm{PE}$, the distribution for PP is more compact. Also the pronounced tendency of PE to unfold inside the CNT is not observed for PP. Notable changes are only observed for the system with of phenyl groups. During the simulation PP passes the phenyl groups to end up in a more stretched configuration leading to higher $R_{\mathrm{g}}$ values close to the center of the CNT.

Due to the final propagation time one has to be careful with interpretation of the absolute numbers. Comparing the scenarios, however, the differences between functional groups and polymer types are becoming obvious. This holds in particular if one notes that PE and PP behave rather similar in water (cf. Fig. 2). 


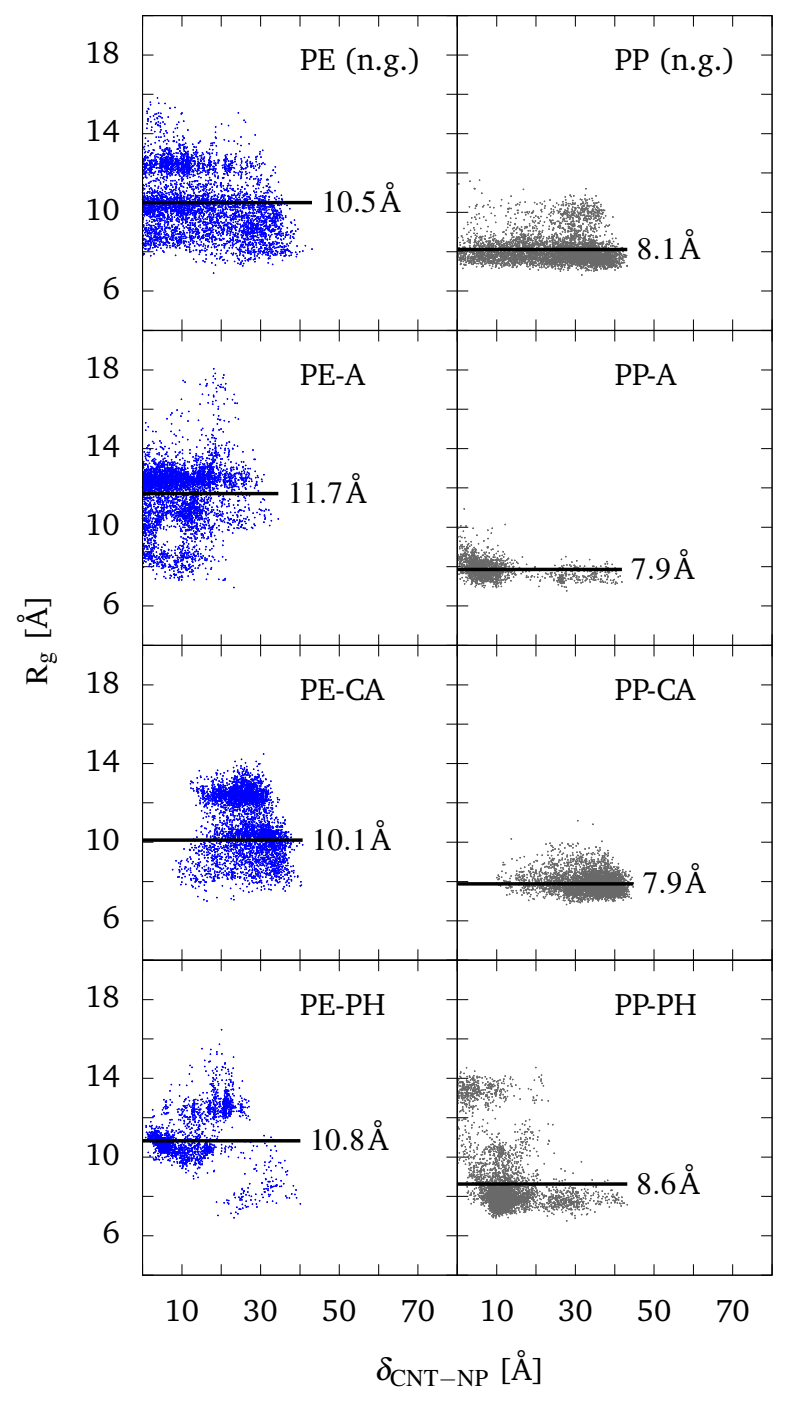

Fig. $6 R_{\mathrm{g}}$ of polymers inside the CNT, depending on the center of mass distance $\delta_{\mathrm{CNT}-\mathrm{NP}}$ between the respective polymer and the CNT. Colors blue and gray relate to PE and PP, respectively. For each polymer, four different systems are investigated, with CNT in absence of functional groups (first row), CNT and alkane chains (second row), CNT and carboxylic acid groups (third row), and CNT and phenyl groups (fourth row) in the system (average $R_{\mathrm{g}}$ - black line).

\section{Conclusions}

Coarse-grained molecular dynamics simulations have been performed to address the interaction of nanoplastics with hydrophobic parts of macromolecular organic soil constituents such as surfaces and functionalized cavities. NP has been modeled by four representative polymers, i.e. the hydrophilic PEO and the hydrophobic PE, PP, and PS. For the hydrophobic surfaces/functionalized cavities a carbon nanotube model has been taken.

First, the behavior of the NP in water has been characterized by means of their radii of gyration. Results were found to be in agreement with Flory theory, thus validating the parametrization of the MARTINI force field for the particular models.

Next, the interaction of the polymers with a bare CNT in water has been investigated. Based on the hydrophobicity/hydrophilicity of the polymers, different adsorption behaviors were observed. PEO as a hydrophilic polymer prefers solvation over adsorption either at the outer wall or inside, whereas the hydrophobic polymers stayed at the CNT, once adsorbed. In general, independent of the radius of gyration, PE showed the strongest interaction with the CNT, followed by PP and PS. For PE and PP, two configurations at the outer CNT wall were observed, which differ in the degree of unfolding. Unfolding increases the contact area with the CNT which leads to a more stable configuration.

The hydrophobicity of the CNT cavity was modified by introducing different hydrophilic and hydrophobic functional groups, i.e. alkane, phenyl and carboxylic acid groups. While PEO has not been much influenced by the cavity and in fact it partly moved out during the simulation, a strong dependence of the behavior on the combination functional group/polymer was observed for the hydrophobic cases. Compared to the solution PE shows the largest increase of the radius of gyration, followed by PP and PS. All three polymers tend to avoid the hydrophilic carboxylic acid groups. On the other hand, they are attracted by the hydrophobic groups and accumulate close to their positions inside the CNT.

Although these results are not unexpected, they can be viewed as a first step towards a mechanistic understanding of a variety of processes, e.g. in environmental soil chemistry, water treatment, and biochemistry. Correlating the present results to soil chemistry points to the critical role of the hydrophobicity in controlling the binding process of NPs in soil. Hydrophobic NPs could have a higher tendency to accumulate in soil and especially soil with high content of hydrophobic soil organic matter (SOM). Furthermore, the results indicate that SOM cavities/voids could play an influential role in accumulation, i.e. immobilization, of NPs in soil. Moreover, the chemical nature of functional groups being present at SOM active surfaces and/or inside SOM cavities/voids plays a role by increasing/decreasing the extent of that accumulation. In a next step refinement of the model can be envisioned, e.g. to include more complex SOM models such as macromolecules 66 77.78 or to incorporate organic pollutants, heavy metals and other compounds bound to the NPs.

It is interesting to note that due to the well defined and unique physicochemical properties of CNTs, they have been used recently in water treatment technologies and especially to remove organic pollutants, e.g., dyes, pharmaceuticals/drugs, pesticides, phenols and aromatic amines. The present results are in accord with the literature in showing that the hydrophobicity of CNTs and modification/decoration of their surfaces with organic functional groups plays an important role in controlling the adsorption/binding/accumulation of organic compounds into outer and inner surfaces of CNTs 79.82 . Therefore, fine tuning and modification for the hydrophobic character of CNTs with organic functional groups could enhance the adsorption capacity of CNTs to remove specific organic pollutants from aqueous solutions and waste water. Thus, the present contribution introduces a very 
fundamental model approaching the idea behind adsorption and removal of organic compounds by CNTs.

\section{Conflicts of interest}

There are no conflicts to declare.

\section{References}

1 K. N. Fotopoulou and H. K. Karapanagioti, The Handbook of Environmental Chemistry, Springer International Publishing, 2017, pp. 71-92.

2 J. Gigault, B. Pedrono, B. Maxit and A. T. Halle, Environmental Science: Nano, 2016, 3, 346-350.

3 Nature Nanotechnology, 2019, 14, 299-299.

4 S. S. Sadri and R. C. Thompson, Marine Pollution Bulletin, 2014, 81, 55-60.

5 M. Zbyszewski, P. L. Corcoran and A. Hockin, Journal of Great Lakes Research, 2014, 40, 288-299.

6 C. M. Free, O. P. Jensen, S. A. Mason, M. Eriksen, N. J. Williamson and B. Boldgiv, Marine Pollution Bulletin, 2014, 85, 156-163.

7 M. R. Gregory, Philosophical Transactions of the Royal Society B: Biological Sciences, 2009, 364, 2013-2025.

8 A. L. Andrady, Marine Pollution Bulletin, 2011, 62, 15961605.

9 A. A. de Souza Machado, W. Kloas, C. Zarfl, S. Hempel and M. C. Rillig, Global Change Biology, 2018, 24, 1405-1416.

10 M. C. Rillig, L. Ziersch and S. Hempel, Scientific Reports, 2017, 7, year.

11 M. Bläsing and W. Amelung, Science of The Total Environment, 2018, 612, 422-435.

12 A. A. Horton, A. Walton, D. J. Spurgeon, E. Lahive and C. Svendsen, Science of The Total Environment, 2017, 586, 127-141.

13 J. Wang, X. Liu, Y. Li, T. Powell, X. Wang, G. Wang and P. Zhang, Science of The Total Environment, 2019, 691, 848857.

14 N. B. Hartmann, S. Rist, J. Bodin, L. H. Jensen, S. N. Schmidt, P. Mayer, A. Meibom and A. Baun, Integrated Environmental Assessment and Management, 2017, 13, 488-493.

15 S. Wagner and T. Reemtsma, 2019, 14, 300-301.

16 O. S. Alimi, J. F. Budarz, L. M. Hernandez and N. Tufenkji, Environmental Science \& Technology, 2018, 52, 1704-1724.

17 A. Käppler, D. Fischer, S. Oberbeckmann, G. Schernewski, M. Labrenz, K.-J. Eichhorn and B. Voit, Analytical and Bioanalytical Chemistry, 2016, 408, 8377-8391.

18 G. Zhang and Y. Liu, Science of The Total Environment, 2018, 642, 12-20.

19 J. N. Möller, M. G. J. Löder and C. Laforsch, Environmental Science \& Technology, 2020, 54, 2078-2090.

20 K. Khosravi-Katuli, E. Prato, G. Lofrano, M. Guida, G. Vale and G. Libralato, Environmental Science and Pollution Research, 2017, 24, 17326-17346.

21 M. F. L. De Volder, S. H. Tawfick, R. H. Baughman and A. J.
Hart, Science, 2013, 339, 535-539.

22 P.-H. Hermes, M.-P. Gabriela, V.-R. Ileana, C. Fusaro, L.V. Fernando, M.-A. Mariana, C. Padilla-Rodríguez and F.-L. Fabián, Green Nanoparticles, Springer International Publishing, Cham, 2020, pp. 77-115.

23 W. Tang, T. Yan, F. Wang, J. Yang, J. Wu, J. Wang, T. Yue and Z. Li, Carbon, 2019, 147, 295-302.

24 V. Erady, R. J. Mascarenhas, A. K. Satpati, A. K. Bhakta, Z. Mekhalif, J. Delhalle and D. A, Microchemical Journal, 2019, 146, 73-82.

25 Y. Bakytkarim, S. Tursynbolat, Q. Zeng, J. Huang and L. Wang, Journal of Electroanalytical Chemistry, 2019, 841, 45-50.

26 Y. Liu, S. Wang, W. Lan and W. Qin, International Journal of Biological Macromolecules, 2019, 121, 1329-1336.

27 M. V. Khodakovskaya, B.-S. Kim, J. N. Kim, M. Alimohammadi, E. Dervishi, T. Mustafa and C. E. Cernigla, Small, 2012, 9, 115-123.

28 X. Ma, J. Geiser-Lee, Y. Deng and A. Kolmakov, Science of The Total Environment, 2010, 408, 3053-3061.

29 P. Begum, R. Ikhtiari, B. Fugetsu, M. Matsuoka, T. Akasaka and F. Watari, Applied Surface Science, 2012, 262, 120-124.

30 H. Hyung and J.-H. Kim, Environmental Science \& Technology, 2008, 42, 4416-4421.

31 M. Ateia, O. G. Apul, Y. Shimizu, A. Muflihah, C. Yoshimura and T. Karanfil, Environmental Science \& Technology, 2017, 51, 7101-7110.

32 M. Engel and B. Chefetz, Advances in Colloid and Interface Science, 2019, 271, 101993.

33 A. Bakir, S. J. Rowland and R. C. Thompson, Marine Pollution Bulletin, 2012, 64, 2782-2789.

34 T. Hüffer and T. Hofmann, Environmental Pollution, 2016, 214, 194-201.

35 S. Seidensticker, P. Grathwohl, J. Lamprecht and C. Zarfl, Environmental Sciences Europe, 2018, 30, year.

36 K. Yang, L. Zhu and B. Xing, Environ. Sci. Technol., 2006, 40, 1855-1861.

37 C.-J. M. Chin, L.-C. Shih, H.-J. Tsai and T.-K. Liu, Carbon, 2007, 45, 1254-1260.

38 W. Chen, L. Duan, L. Wang and D. Zhu, Environ. Sci. Technol., 2008, 42, 6862-6868.

39 D. Petrov, D. Tunega, M. H. Gerzabek and C. Oostenbrink, Environ. Sci. Technol., 2017, 51, 5414-5424.

40 A. A. Ahmed, S. Thiele-Bruhn, S. G. Aziz, R. H. Hilal, S. A. Elroby, A. O. Al-Youbi, P. Leinweber and O. Kühn, Sci. Total Environ., 2015, 508, 276-87.

41 E. Slonkina and A. B. Kolomeisky, The Journal of Chemical Physics, 2003, 118, 7112-7118.

42 H.-P. Hsu and P. Grassberger, The Journal of Chemical Physics, 2004, 120, 2034-2041.

43 W. Reisner, K. J. Morton, R. Riehn, Y. M. Wang, Z. Yu, M. Rosen, J. C. Sturm, S. Y. Chou, E. Frey and R. H. Austin, Physical Review Letters, 2005, 94, year.

44 W. Sparreboom, A. van den Berg and J. C. T. Eijkel, Nature Nanotechnology, 2009, 4, 713-720. 
45 G. O. Ibáñez-García and P. Goldstein-Menache, Soft Matter, 2012, 8, 8666.

46 B.-Y. Ha and Y. Jung, Soft Matter, 2015, 11, 2333-2352.

47 M. P. Taylor, Macromolecules, 2017, 50, 6967-6976.

48 M. Rubinstein and R. Colby, Polymer Physics, OUP Oxford, 2003.

49 Q. Cao and M. Bachmann, Soft Matter, 2017, 13, 600-607.

50 S. Jun and A. Wright, Nature Reviews Microbiology, 2010, 8, 600-607.

51 F. Müller-Plathe, ChemPhysChem, 2002, 3, 754-769.

52 J. Baschnagel, K. Binder, P. Doruker, A. A. Gusev, O. Hahn, K. Kremer, W. L. Mattice, F. Müller-Plathe, M. Murat, W. Paul, S. Santos, U. W. Suter and V. Tries, Viscoelasticity, Atomistic Models, Statistical Chemistry, Springer Berlin Heidelberg, Berlin, Heidelberg, 2000, vol. 152, pp. 41-156.

53 J. Zhao, J.-W. Jiang, L. Wang, W. Guo and T. Rabczuk, Journal of the Mechanics and Physics of Solids, 2014, 71, 197-218.

54 S. Cranford and M. Buehler, Multiscale Modeling, CRC Press, 2010, pp. 13-34.

55 R. Geyer, J. R. Jambeck and K. L. Law, Science Advances, 2017, 3, e1700782.

56 S. Piehl, A. Leibner, M. G. J. Löder, R. Dris, C. Bogner and C. Laforsch, Scientific Reports, 2018, 8, year.

57 F. A. E. Lots, P. Behrens, M. G. Vijver, A. A. Horton and T. Bosker, Marine Pollution Bulletin, 2017, 123, 219-226.

58 D. He, Y. Luo, S. Lu, M. Liu, Y. Song and L. Lei, TrAC Trends Anal. Chem., 2018, 109, 163-172.

59 J.-J. Guo, X.-P. Huang, L. Xiang, Y.-Z. Wang, Y.-W. Li, H. Li, Q.Y. Cai, C.-H. Mo and M.-H. Wong, Environment International, 2020, 137, 105263.

60 S. J. Marrink, H. J. Risselada, S. Yefimov, D. P. Tieleman and A. H. de Vries, The Journal of Physical Chemistry B, 2007, 111, 7812-7824.

61 F. Grunewald, G. Rossi, A. H. de Vries, S. J. Marrink and L. Monticelli, The Journal of Physical Chemistry B, 2018, 122, 7436-7449.

62 E. Panizon, D. Bochicchio, L. Monticelli and G. Rossi, The Journal of Physical Chemistry B, 2015, 119, 8209-8216.

63 G. Rossi, L. Monticelli, S. R. Puisto, I. Vattulainen and T. AlaNissila, Soft Matter, 2011, 7, 698-708.
64 L. Martínez, R. Andrade, E. G. Birgin and J. M. Martínez, Journal of Computational Chemistry, 2009, 30, 2157-2164.

65 M. Vögele, J. Köfinger and G. Hummer, Faraday Discussions, 2018, 209, 341-358.

66 H. R. Schulten and M. Schnitzer, Naturwissenschaften, 1993, 80, 29-30.

67 J. Kubicki and C. Trout, Geochemical and Hydrological Reactivity of Heavy Metals in Soils, CRC Press, 2003.

68 A. J. A. Aquino, D. Tunega, G. Haberhauer, M. H. Gerzabek and H. Lischka, Eur. J. Soil Sci., 2007, 58, 889-899.

69 A. J. A. Aquino, D. Tunega, H. Pašalić, G. E. Schaumann, G. Haberhauer, M. H. Gerzabek and H. Lischka, Environ. Sci. Technol., 2011, 45, 8411-8419.

70 A. A. Ahmed, O. Kühn and P. Leinweber, Sci. Total Environ., 2012, 441, 151-158.

71 A. A. Ahmed, O. Kühn, S. G. Aziz, R. H. Hilal and P. Leinweber, Sci. Total Environ., 2014, 476-477, 98-106.

72 Lindahl, , Abraham, Hess and V. D. Spoel, GROMACS 2020.3 Manual, Zenodo, 2020.

73 G. Bussi, D. Donadio and M. Parrinello, The Journal of Chemical Physics, 2007, 126, 014101.

74 S. J. Marrink, A. H. de Vries and A. E. Mark, J. Phys. Chem. B, 2004, 108, 750-760.

75 S. J. Marrink, X. Periole, D. P. Tieleman and A. H. de Vries, Phys. Chem. Chem. Phys., 2010, 12, 2254.

76 W. Humphrey, A. Dalke and K. Schulten, Journal of Molecular Graphics, 1996, 14, 33-38.

77 H.-R. Schulten and P. Leinweber, Biol. Fertil. Soils, 2000, 30, 399-432.

78 H. Feng, H. Zhang, H. Cao, Y. Sun, A. Zhang and J. Fu, Environ. Sci. Technol., 2018, 52, 14228-14234.

79 V. K. Gupta, R. Kumar, A. Nayak, T. A. Saleh and M. Barakat, Advances in Colloid and Interface Science, 2013, 193-194, 24 34.

80 W. Wu, K. Yang, W. Chen, W. Wang, J. Zhang, D. Lin and B. Xing, Water Research, 2016, 88, 492-501.

81 J.-G. Yu, X.-H. Zhao, H. Yang, X.-H. Chen, Q. Yang, L.-Y. Yu, J.-H. Jiang and X.-Q. Chen, Science of The Total Environment, 2014, 482-483, 241-251.

82 X. Qu, P. J. Alvarez and Q. Li, Water Research, 2013, 47, 39313946. 


\title{
Supplementary Information (SI)
}

\section{Coarse-grained molecular dynamics simulations of nanoplastics interacting with a hydrophobic environment in aqueous solution}

\author{
Lorenz F. Dettmann ${ }^{a}$, Oliver Kühn ${ }^{a b}$, and Ashour A. Ahmed ${ }^{a b *}$
}

\author{
${ }^{a}$ University of Rostock, Institute of Physics, Albert-Einstein-Str. 23-24, D-18059 Rostock, Germany. \\ E-mail: ashour.ahmed@uni-rostock.de \\ ${ }^{b}$ University of Rostock, Department of Life, Light and Matter (LLM), Albert-Einstein-Str. 25, D-18059 Rostock, Ger- \\ many
}

\section{Diffusion of coarse-grained water through CNTs}

The goal of the present investigation was to find a suitable CNT structure for studying interactions and movements of polymers inside and outside the CNT. Therefore, the corresponding water particles in presence of the CNT should have a relatively constant diffusion to make the movement of the NP smooth. The movement of the water particles was investigated by calculating their mean-squared-displacements (MSDs):

$$
\left\langle\Delta \mathbf{r}(t)^{2}\right\rangle=\frac{1}{N} \sum_{i=1}^{N}\left(\mathbf{r}_{i}(t)-\mathbf{r}_{i}(0)\right)^{2}
$$

A relatively congruent and constant rise of the calculated MSDs for the water inside a CNT indicates a constant diffusion of the water particles. The whole set of the CNTs had three different diameters: $15 \AA, 20 \AA$ and $30 \AA$. . Furthermore for each diameter, three different lengths were used: $50 \AA, 100 \AA$ and $125 \AA$. In total, this results in nine CNT models. Each model is marked with one number (see Tab. S1). Periodic boundary conditions were applied in each Cartesian dimension. The initial box dimensions were designed so that the tubes have a distance of $50 \AA$ in $x$ - and $y$-direction and at least $50 \AA$ in $z$-direction to their periodic image. The boxes were filled with water which has an initial density of approximately $1 \mathrm{~g} \mathrm{~cm}^{-3}$.

First, for every system, an energy minimization was performed. With the resulting structures, an NPT simulation for 100 ns was done, to adjust the box dimensions. Finally, for each system, the MSD was calculated by NVT simulation, with a simulation time of $500 \mathrm{~ns}$. The box dimensions of the NPT simulations were averaged over the whole time and applied to the $N V T$ systems. MSDs of water particles inside the CNTs were calculated using the VMD RMSD tool. For water, an antifreeze particle concentration of $A F=15 \%$ was applied. This concentration was sufficient to prevent the freezing of water in most of the models. Due to the antifreeze particles, the density of the systems changed to around $0.86 \mathrm{~g} \mathrm{~cm}^{-3}$ after equilibration.

Since the MSD can just be calculated for a fixed particle selection, one has to make sure that only water in the inner region is included into the analysis. Therefore, the following procedure was applied: First, one chooses a snapshot of the trajectory and selects a random unbiased set of water particles in the middle of the inner CNT, for this snapshot. This selection is restricted in a volume that goes $5 \AA$ in the positive and negative $z$-direction from the middle of the CNT and to its inner walls (see Fig. S1). Then, from the chosen snapshot on, one starts the trajectory. Thereby, the selection of water particles is moving inside the CNT, until one of the particles is going to leave the CNT. This new snapshot would mark the end of the analysis interval. For the resulting time span, the MSD is calculated just for this selection. For one CNT, this analysis is repeated six times, starting at six different snapshots of the corresponding $N V T$ trajectory. It has to be noted that due to the random choice and the different diameters of the CNT that the number of particles in the selection is varying. The resulting MSDs are shown in Fig. S2. Furthermore, the MSD of bulk water is plotted in every diagram (black line). The individual calculated curves for one CNT should be similar as possible, to indicate a relatively constant diffusion of the water particles.

In overall, the fluctuations of the MSDs are strong, compared to the MSD for bulk water. The main reason for this might be the relatively low number of water particles used for the calculation. Model 8 and 9 show sufficient congruence between the individual MSDs, as well as a relatively constant rise. Finally, after considering these two models, the CNT of model 8 was chosen for the investigation with NPs, because it would require a smaller simulation box, due to its smaller length. 
Table S1: Detailed Information about the model systems, with $d$ and $l$ being the diameter and the length of the CNT, followed by the box dimensions. The other columns denote the number of water particles, number of carbon atoms in the atomistic representation, number of beads in the coarse-grained system, number of rings of the CNT, ring size (how many beads in one ring) and the bond length between two beads.

\begin{tabular}{cccccccccc}
\hline model & $d[\AA]$ & $l[\AA]$ & $\operatorname{Box}\left[\AA^{3}\right]$ & $N_{W}$ & $N_{C}(\mathrm{aa})$ & $N_{b}(\mathrm{CG})$ & $N_{R}(\mathrm{CG})$ & $R s(\mathrm{CG})$ & $B l[\AA]$ \\
\hline 1 & 15 & 50 & $65 \times 65 \times 175$ & 5960 & 960 & 130 & 13 & 10 & 4.7 \\
2 & 15 & 100 & $65 \times 65 \times 175$ & 5960 & 1920 & 260 & 26 & 10 & 4.7 \\
3 & 15 & 125 & $65 \times 65 \times 175$ & 5960 & 2400 & 320 & 32 & 10 & 4.7 \\
\hline 4 & 20 & 50 & $70 \times 70 \times 175$ & 6900 & 1248 & 182 & 13 & 14 & 4.7 \\
5 & 20 & 100 & $70 \times 70 \times 175$ & 6900 & 2496 & 364 & 26 & 14 & 4.7 \\
6 & 20 & 125 & $70 \times 70 \times 175$ & 6900 & 3120 & 448 & 32 & 14 & 4.7 \\
\hline 7 & 30 & 50 & $80 \times 80 \times 175$ & 9100 & 1872 & 260 & 13 & 20 & 4.7 \\
8 & 30 & 100 & $80 \times 80 \times 175$ & 9100 & 3744 & 520 & 26 & 20 & 4.7 \\
9 & 30 & 125 & $80 \times 80 \times 175$ & 9100 & 4680 & 640 & 32 & 20 & 4.7 \\
\hline
\end{tabular}

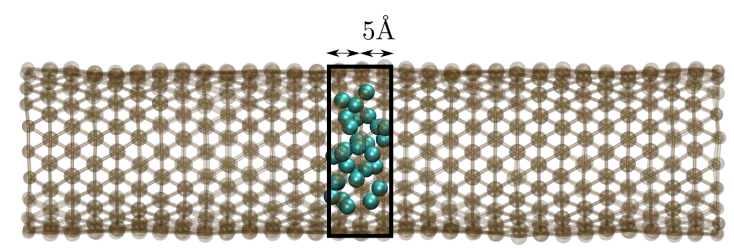

(a)

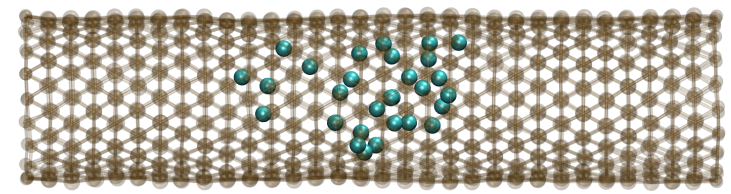

(b)

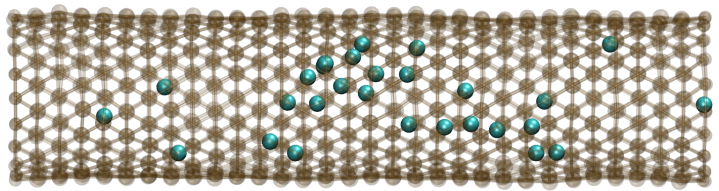

(c)

Figure S1: Visualization of a certain selection of water particles and their time evolution for model 9. From such a trajectory, one MSD is calculated for the corresponding CNT and repeated six times at different starting points in the whole simulation and with a new particle selection. Picture (a) shows the trajectory after $0 \mathrm{~ns}$, picture (b) after $0.18 \mathrm{~ns}$ and picture (c) after $3.56 \mathrm{~ns}$ of the analysis interval. 

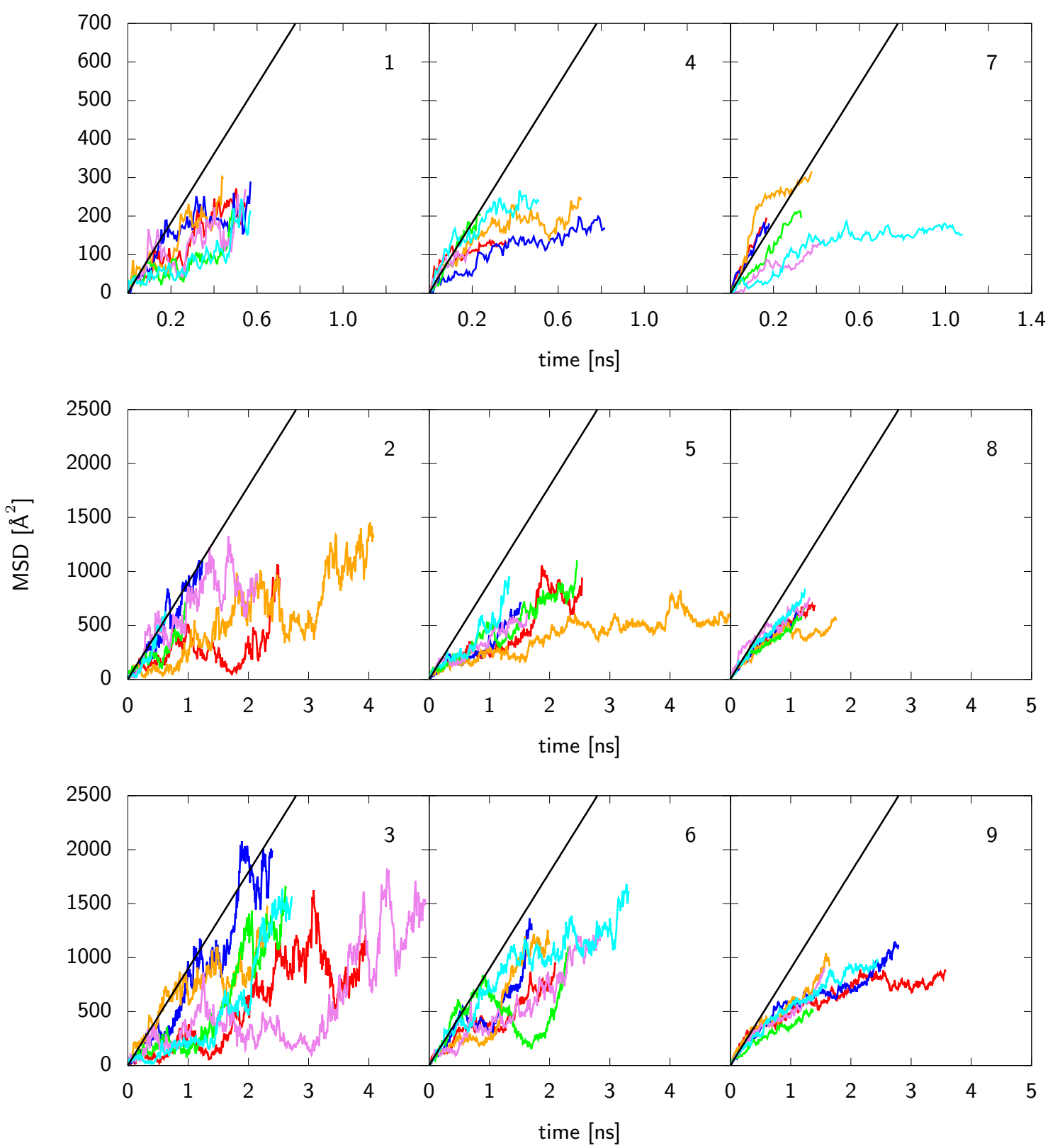

Figure S2: MSD curves for the models 1 to 9 . In each system, the MSD was calculated six times, resulting in six MSDs with the colors blue, red, orange, cyan, green, and violet. The black lines represent the MSD of bulk water from another model system. 


\section{Interaction energies and radial distribution functions between NPs and water}

Table S2: Information about the individual polymer types regarding their coarse-grained representation. Bond lengths relate to the equilibrium distance between two neighboring beads. For PS the bond length applies to every bond of the polymer. The bead names and their corresponding LJ parameters are defined in the MARTINI force field. The beads consist of the listed atoms. One phenyl ring of PS is represented by three STY beads, which are identical.

\begin{tabular}{lllll}
\hline Polymer & PEO & PE & PP & PS \\
\hline Bond length (CG) & $0.322 \mathrm{~nm}($ EO-EO), 0.280 nm (SP2-EO) & $0.460 \mathrm{~nm}$ & $0.298 \mathrm{~nm}$ & $0.270 \mathrm{~nm}$ \\
Bead names & $\mathrm{SP} 2, \mathrm{EO}$ & $\mathrm{C} 1$ & $\mathrm{SC} 1$ & $\mathrm{STY}, \mathrm{SCY}$ \\
Atoms & $\mathrm{OHCH}_{2}, \mathrm{CH}_{2} \mathrm{OCH}_{2}$ & $4 \times \mathrm{CH}_{2}$ & $\mathrm{CH}_{2} \mathrm{CHCH}_{3}$ & $\mathrm{CHC}(\mathrm{H}), \mathrm{CH}_{2} \mathrm{CH}$ \\
Mass in u (1 Bead) & 31,44 & 56 & 42 & 26,27 \\
\hline
\end{tabular}
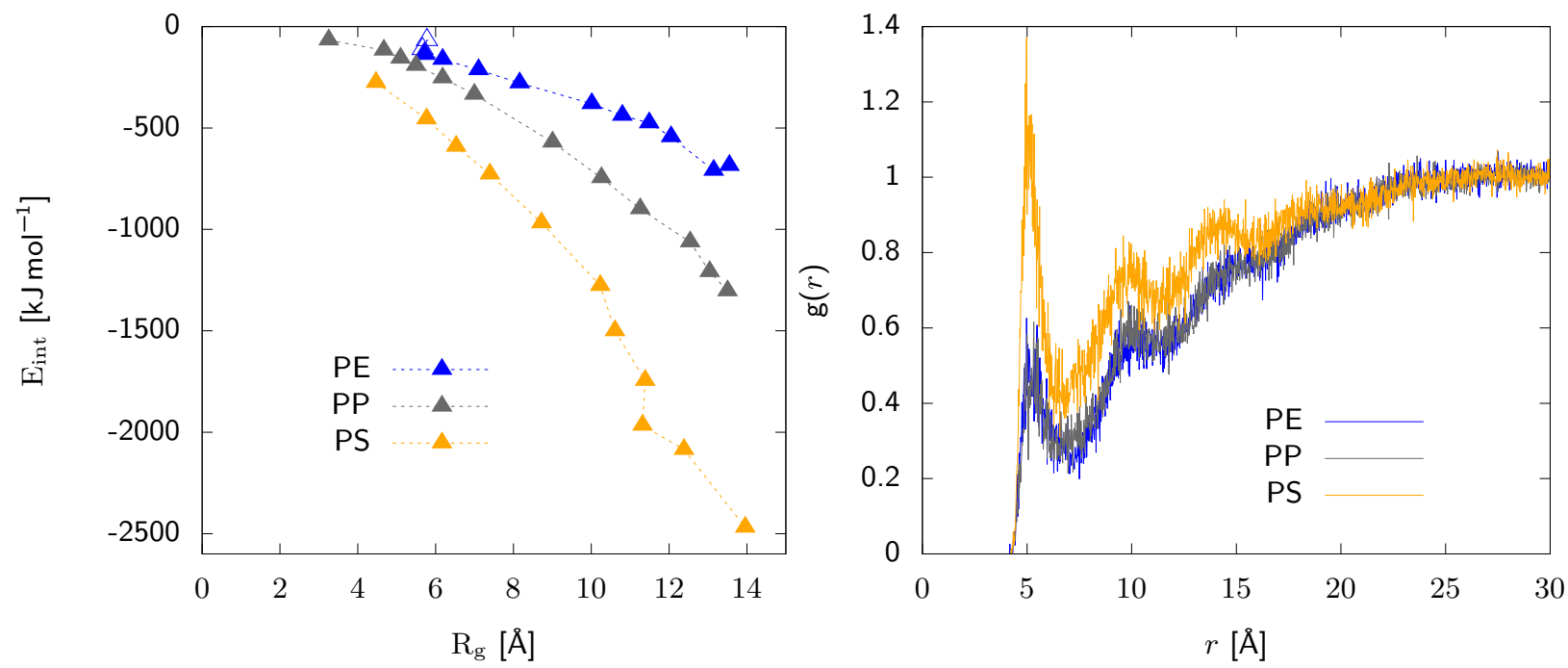

Figure S3: Interaction energy between NPs and water depending on their average $R_{g}$.

Figure S4: Radial distribution function (RDF) of water to NPs, having an $R_{g}$ of around $10 \AA$. For calculating the RDFs, one bead was selected at the surface of each NP particle.

From Fig. S3 we conclude that PS has a low hydrophobicity compared to PE and PP, due to the relatively strong interaction with water. A similar result is given by the high peak of the RDF for PS to water, shown in Fig. S4. However, it should be noted that the coarse-grained potential for PS is not retaining its tacticity, so that the phenyl groups can move freely around the alkane chain. It was therefore observed, that the phenyl groups are mostly located towards water. This effect might underestimate the hydrophobicity of PS.

\section{Snapshots for PE and interaction energies for PP \& PS}

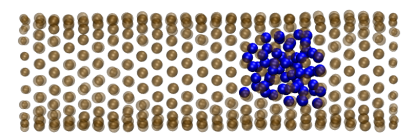

(a)

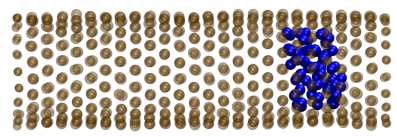

(b)

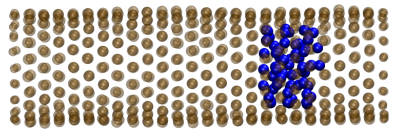

(c)

Figure S5: Snapshots from the $N V T$ simulation with PE initially placed inside the CNT $\left(\mathrm{R}_{\mathrm{g}}=9 \AA\right)$. (a) shows the initial position, (b) the trajectory after $250 \mathrm{~ns}$ and (c) the trajectory after $500 \mathrm{~ns}$. PE doesn't leave the CNT, indicating a generally high stability of adsorbed configurations. 
Additional information about the starting configurations for the models with polymers placed outside the CNT can be summarized as follows: Some polymers did not interact with the CNT. In such cases, the simulation is repeated with slightly different starting configurations or continued, until an interaction is observed. So, the first starting configuration can vary, depending on the course of the previous simulation. Also, if e.g. the polymer adsorbs to fast at the outer wall, the time window for the interaction energy recording in solution may be too short, which might result in a larger error for the calculated energy, compared to a longer solution phase period. In such a case, the simulation is repeated with the only change that the polymer is placed more far away from the outer wall of the CNT (e.g. with a distance of $40 \AA$ ). It was also observed that a polymer initially placed in solution, was going inside the CNT during the simulation. Then, the simulation is repeated with a higher distance to the CNT. The course of the simulations is strongly depending on the starting conditions, e.g. the initial velocities of the water particles. Relevant for this investigation are the time intervals in the simulations, when the polymers are in bulk water, adsorbed at the outer wall or inside the CNT. These time intervals should be relatively large, so that the calculated interaction energies have a lower error.

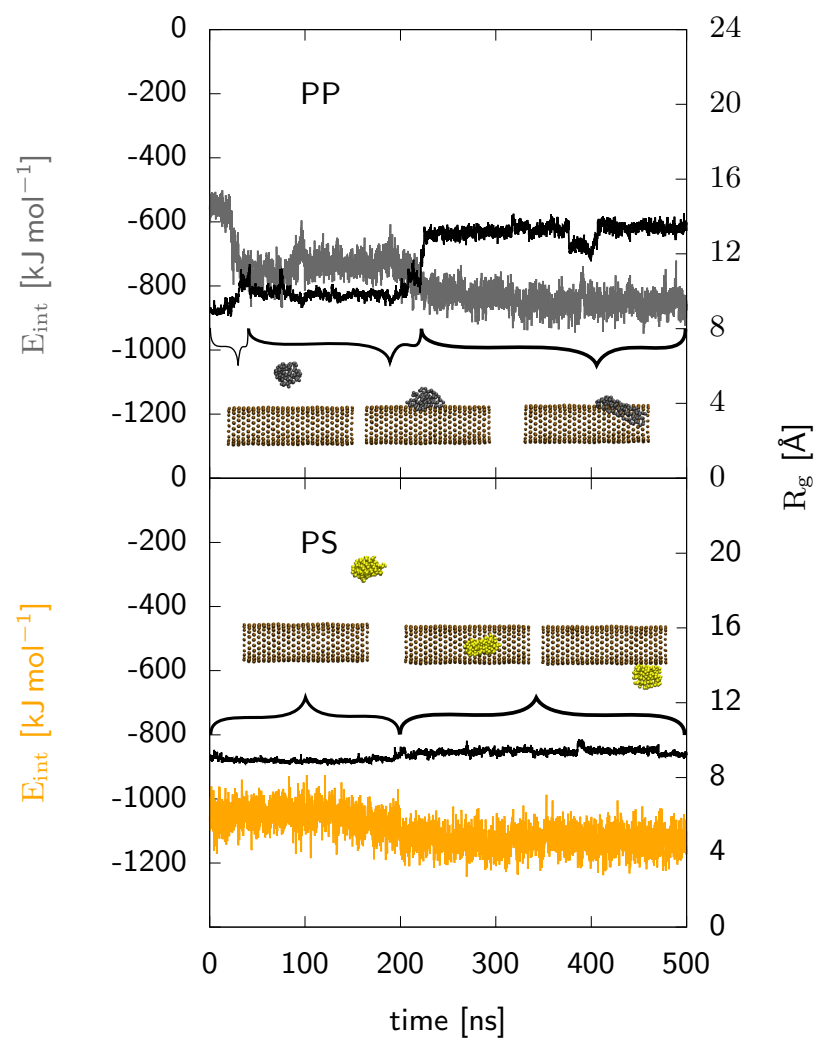

Figure S6: Interaction energies in the course of the $N V T$ simulations for PP (gray) and PS (orange) with initially $R_{\mathrm{g}}=9 \AA$. For both polymers, the lines relate to the simulations with the polymer chains outside the CNT. The interaction energy between the respective chain and the rest of the system is considered. Furthermore, $\mathrm{R}_{\mathrm{g}}$ (black) is plotted for both polymer types. 
IV. Lateral position densities and radii of gyration for PEO \& PS inside the CNTs

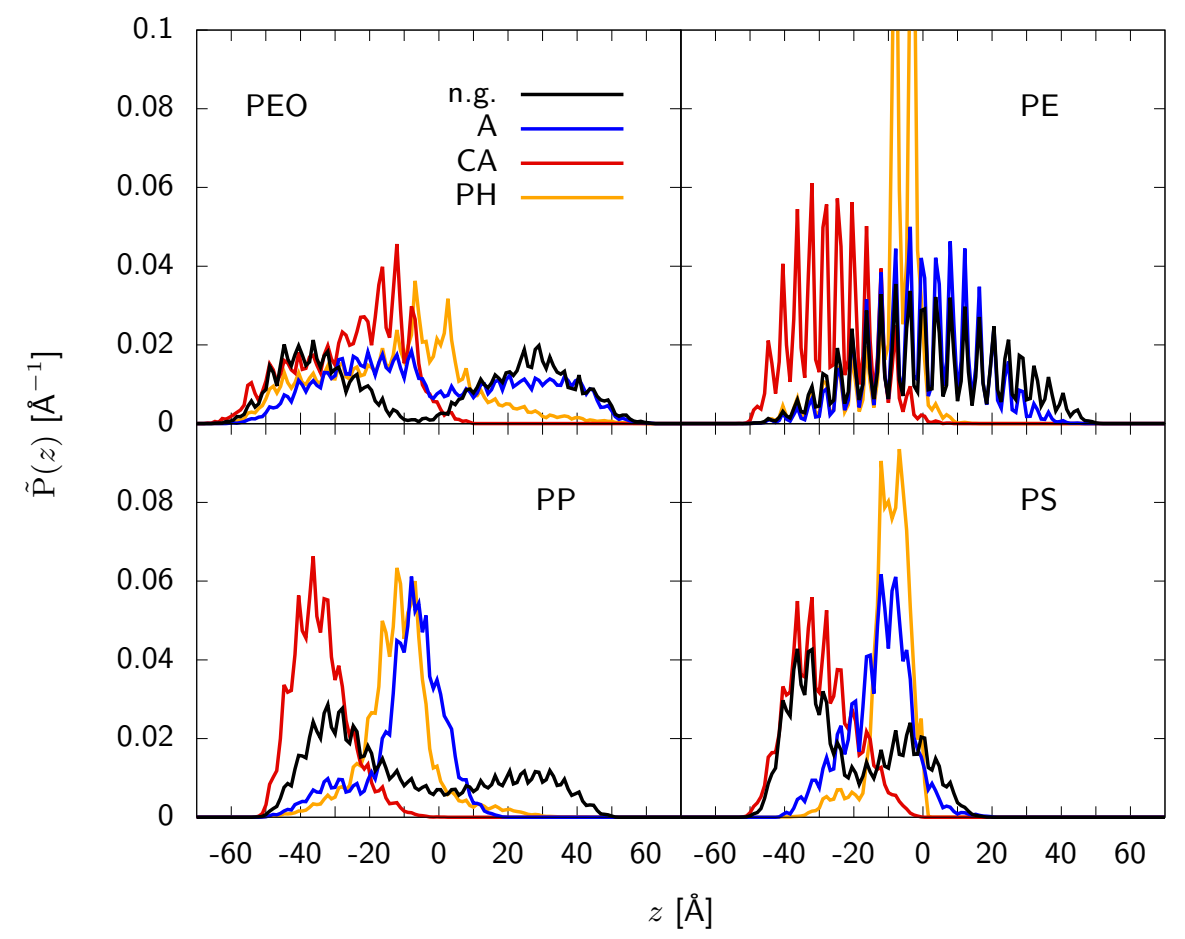

Figure S7: Normalized partial densities, $\tilde{P}(z)$, for different polymers being (mostly) inside the CNT $(-50 \AA<z<50 \AA)$, depending on their position $z$. Here, densities are not symmetrized, like in Fig. 5 of the main text. $z=0$ denotes the center of mass of the CNT, or rather the position of functional groups, if present. The polymers starting configurations were in the range of negative $z$ values. In these diagrams one can see that in some cases of present functional groups, it is possible that the polymer penetrates into the range of positive $z$ values (e.g. PEO-A, or PE-A). Note that for PE-PH the two maxima which are out of the scale reach $\sim 0.16 \AA^{-1}$.

For the functional groups, the particle/bead C1 from the MARTINI force field was used for alkane chains, P3 for carboxylic acid groups and SC5 for phenyl groups. 


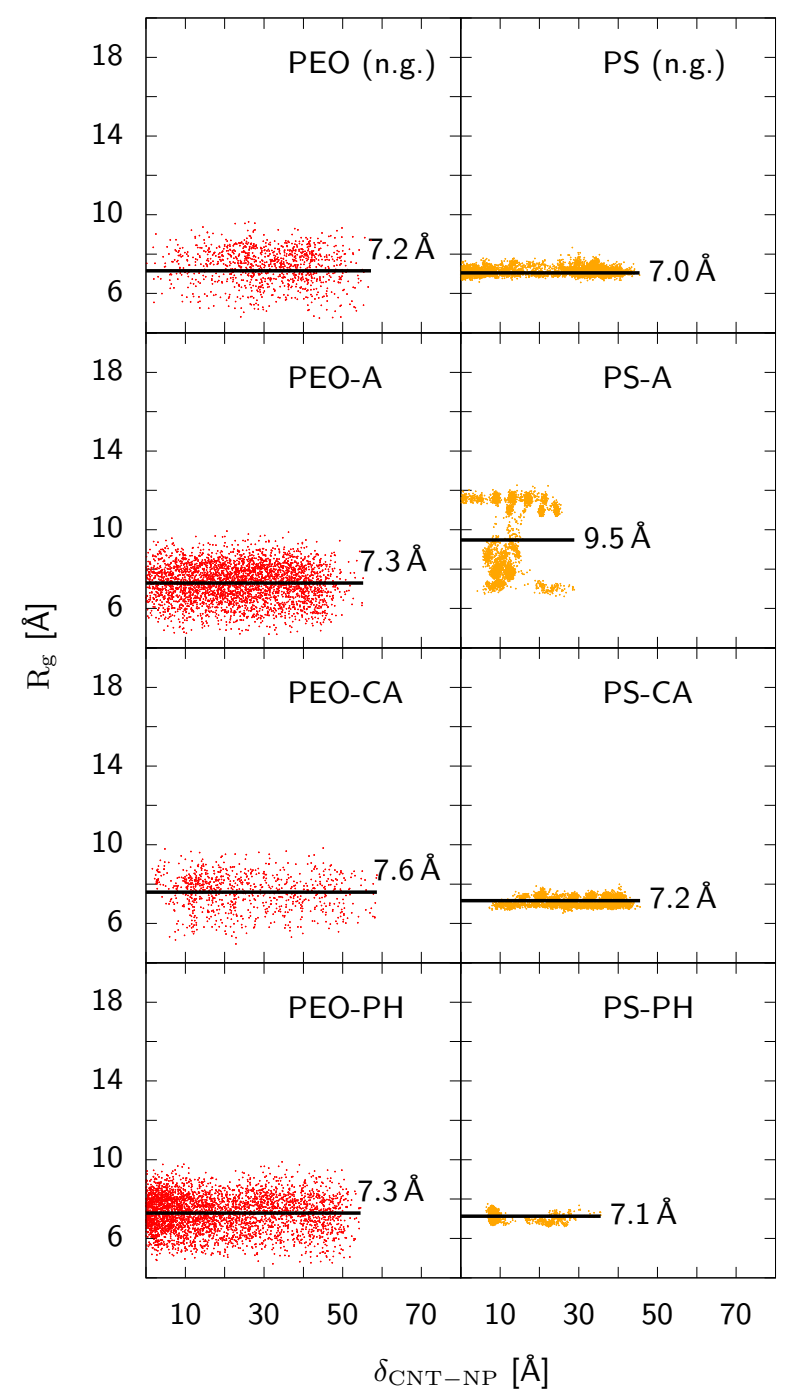

Figure S8: $\mathrm{R}_{\mathrm{g}}$ of polymer chains inside the CNT, depending on the center of mass distance $\delta_{\mathrm{CNT}-\mathrm{P}}$ between the respective polymer and the CNT. Colors red and orange relate to the polymer types PEO and PS, respectively. The radius of gyration for $\mathrm{PEO}$ is not significantly influenced by present functional groups, visible in the very similar distributions of $R_{g}$. Only the sample density (number of data points) for the cases with bare CNT and attached carboxylic acid groups is smaller, because the polymer left the CNT at earlier times. PS on the other hand shows a higher distribution for the case with alkane chains, due to an unfolding of the polymer. In all the other models, $\mathrm{R}_{\mathrm{g}}$ remains relatively constant, underlining the rigidity of PS. 

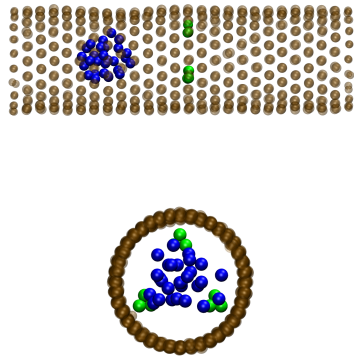

(a)
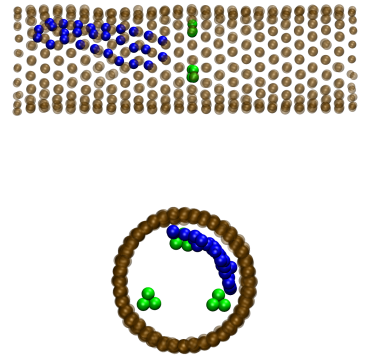

(b)
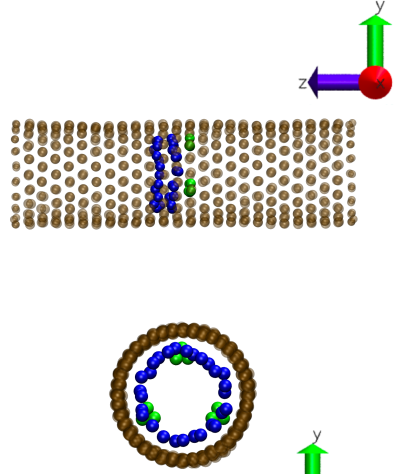

(c)

Figure S9: Snapshots from the NVT simulation of PE (blue) being inside the CNT (brown) with phenyl groups (green). Two pictures in one figure correspond to the side and front view on the system. (a) shows the initial position, (b) shows the trajectory after $100 \mathrm{~ns}$, and (c) the trajectory after $308 \mathrm{~ns}$. In this simulation, PE folds itself to a ring structure right next to the phenyl groups. For a better visualization, water particles are left out. 